\title{
电子束熔炼制备太阳能级多晶硅的研究现状与发展趋势
}

\author{
谭 毅 $^{1,2}$, 石 爽 ${ }^{1,2}$, 姜大川 1,2
}

(大连理工大学 1. 材料科学与工程学院; 2. 辽宁省太阳能光伏系统重点实验室, 大连 116024)

摘 要: 电子束熔炼具有高能量密度、高真空度等优点, 能够有效地去除硅中的挥发性杂质, 使其在制备太阳能级 多晶硅材料方面具有巨大的优势和广阔的应用前景，目前已经实现了产业化应用，成为冶金法制备太阳能级硅材 料的关键环节之一。本文在阐述挥发性杂质去除的热力学原理的基础上，对其去除效果和去除机制进行了总结。同 时, 针对电子束熔炼技术目前存在的问题, 结合作者在这些方面的探索, 从数值模拟、节能型熔炼方式以及与定向 凝固技术的耦合等角度对现阶段的研究重点进行了综述，并对其未来的发展趋势进行了展望。

关 键 词: 电子束熔炼; 多晶硅; 挥发性杂质; 综述

中图分类号: TF134; TF89 文献标识码: A

\section{Progress in Research and Development of Solar-grade Silicon Preparation by Electron Beam Melting}

\author{
TAN Yi ${ }^{1,2}$, SHI Shuang ${ }^{1,2}$, JIANG Da-Chuan ${ }^{1,2}$
}

\begin{abstract}
(1. School of Materials Science and Engineering, Dalian University of Technology, Dalian 116024, China; 2. Key Laboratory
\end{abstract} for Solar Energy Photovoltaic System of Liaoning Province, Dalian 116024, China)

\begin{abstract}
Electron beam melting is an effective method to remove volatile impurities in silicon due to its characteristics of high energy density and high vacuum degree, which has huge advantages and wide application prospects in preparation of solar-grade silicon. Currently it has been successfully applied in industry and become one of the key procedures for preparation of solar-grade silicon by metallurgical route. Based on the thermodynamic principle of the volatile impurities removal, the removal efficiency and mechanism are summarized in this paper. According to current problems of this technology combined with our own experience, the emphasis of current research is reviewed from the points of numerical simulation, energy-saving melting technology and coupling with directional solidification technology. The future development direction of this technology is also proposed.
\end{abstract}

Key words: electron beam melting; solar-grade silicon; volatile impurities; review

太阳能作为一种可再生能源，具有储量巨大、 清洁无污染、来源稳定，且不受地域限制的优点，是 解决未来能源紧缺的理想选择，已成为世界各国广 泛研究和推广使用的重要能源。近年来, 光伏发电 产业快速发展 ${ }^{[1]}$, 根据国际能源署的预测, 到 2050 年 全球光伏发电累计装机容量将达到 $3000 \mathrm{GW}$, 约占
全球电力生产的 $11 \%$, 发展潜力巨大。因此, 世界 各国都高度重视以光伏发电为代表的新能源产业, 我国也明确提出大力发展光伏产业, 并将以其为代 表的新能源上升到国家战略高度。

光伏产业的发展将大幅度增加对太阳能电池材 料的需求, 作为太阳能电池的主要原材料, 晶硅材

收稿日期: 2014-12-22; 收到修改稿日期：2015-02-09

基金项目：国家自然科学基金一云南联合基金(U1137601); 高等学校博士学科点专项科研基金(20130041110004) National Natural Science Foundation of China(U1137601); Specialized Research Fund for the Doctoral Program of Higher Education(20130041110004)

作者简介：谭 毅(1961-), 男, 博士, 教授.E-mail: tanyi@dlut.edu.cn 
料占据了全球光伏市场 $80 \%$ 以上的市场份额 ${ }^{[2-4]}$ 。其 中, 多晶硅虽然效率略低于单晶硅, 但其具有低成 本的优势, 成为最广泛使用的太阳能电池材料。太 阳能电池的转换效率取决于硅的纯度 ${ }^{[5-7]}$, 通常认 为硅材料的纯度需要达到 $99.9999 \%$, 才能满足太阳 能电池的使用要求, 称其为太阳能级多晶硅 (SoG-Si)。硅中的主要杂质为 P、B 和金属杂质, 这 些杂质会严重影响硅材料的电学性能, 需要被去除 到很低的程度。冶金法就是根据这些杂质元素与硅 的物理性质差异, 采用不同的技术依次将其去除的 集成方法，包括介质熔炼、定向凝固、电子束熔炼 等, 这种方法具有成本低、无污染、可大规模生产 等优点, 成为当前研究的热点 ${ }^{[8-11]}$ 。

电子束熔炼具有高能量密度和高真空度等优点, 在金属及合金的熔炼和精炼领域具有广泛的应用 ${ }^{[12]}$ 。 1907 年, Von Pirani 申请了一项专利, 标志着电子束 技术的诞生 ${ }^{[13]}$, 然而当时的真空技术落后, 尚不足 以将电子束技术应用到实际生产中; 在随后的几十 年里, 真空技术不断发展, 有了长足的进步, Temescal 冶金公司在 1957 年实现了电子束技术的工 业化应用。20 世纪 80 年代, Casenave 等 ${ }^{[14]}$ 首次使用 电子束技术提纯了多晶带硅材料; 20 世纪 90 年代, 东京大学 Ikeda 等 ${ }^{[15]}$ 使用电子束熔炼方式提纯了多 晶硅材料, 并研究了杂质的去除机制; 随后, 日本 JFE 开展了冶金法制备太阳能级多晶硅的研究与应 用, 并将电子束熔炼纳入到冶金法的流程中 ${ }^{[16-17]}$ 。 目前, 电子束熔炼已成为冶金法制备太阳能级多晶 硅材料的关键环节, 并已在产业上得到了应用。

本文在介绍硅中挥发性杂质去除的热力学原理 的基础上, 对电子束熔炼条件下杂质的去除效果、 去除机制以及现阶段的研究热点进行了综述, 指出 了目前存在的问题, 并着重分析了这些问题的解决 对策，阐明了电子束技术应用于多晶硅提纯领域的 技术优势, 并对下一阶段电子束熔炼提纯多晶硅技 术的研究重点和发展前景进行了展望。

\section{1 挥发性杂质去除的热力学原理}

具有多杂质的基体金属或者合金在高温、高真空 的条件下, 饱和蒸汽压大的元素挥发性大于饱和蒸汽 压小的元素, 这是真空蒸发提纯的理论依据 ${ }^{[18-19]}$ 。根据 Clausius-Clapeyron 方程, 纯物质的饱和蒸汽压只是 温度的函数

$$
\lg P_{i}^{0}=\frac{A}{T}+B \lg T+C T+D
$$

式中 $A 、 B 、 C 、 D$ 是饱和蒸气压常数。在硅的熔炼
温度范围内, 各种元素的饱和蒸气压随温度的升高 而增大。除 B 元素外, 硅中主要杂质元素的饱和蒸 气压都大于电子束熔炼真空室的气压, 可以从硅熔 体表面蒸发进入气相中。

然而, 在熔炼过程中, 杂质从硅熔体表面蒸发, 同时硅基体在此条件下也会蒸发, 因此杂质最终被 去除还是被浓缩取决于杂质与硅的蒸发之比。杂质 在硅熔体中的蒸发能力可用蒸发系数 $\beta$ 来表征

$$
\beta=\sqrt{\frac{M_{\mathrm{Si}}}{M_{i}}} \frac{\gamma_{i}^{0} P_{i}^{0}}{P_{\mathrm{Si}}^{0}}
$$

其中, $M_{\mathrm{si}}$ 和 $M_{i}$ 分别是硅和杂质元素的摩尔质量; $\gamma_{i}^{0}$ 是杂质元素在硅熔体中的活度系数; $P_{\mathrm{Si}}^{0}$ 和 $P_{i}^{0}$ 分 别是硅和杂质元素的饱和蒸气压。根据 $\beta$ 值, 杂质 在硅中的蒸发分为三种情况: (1)当 $\beta<1$ 时, 杂质的 蒸发能力小于硅, 熔炼后杂质不但不会被去除反而 被浓缩; (2)当 $\beta=1$ 时, 硅与杂质的蒸发能力相等, 即 硅与杂质按照熔体初始成分同步挥发, 熔体的成分 在熔炼前后不会变化, 所以杂质含量不变; (3)当 $\beta>1$ 时, 杂质的蒸发能力大于硅, 熔炼后杂质含量 减少, 可以被去除。 $\beta$ 值越大, 杂质越容易被去除, 通常认为 $\beta>10$ 时杂质可以被有效去除。硅中主要 杂质的蒸发系数与温度的关系如图 1 所示, $\mathrm{P} 、 \mathrm{Al}$ 、 $\mathrm{Ca}$ 等元素的蒸发系数均大于 1 , 在高温真空的条件 下可以被蒸发去除。随着温度的升高, 硅的蒸发能力 显著增加, 因此杂质的蒸发系数逐渐减小。Fe、B 两 种元素的蒸发系数小于 1 , 无法通过这种方式去除。

\section{2 杂质去除效果}

电子束熔炼是利用高能量密度的电子束轰击材 料表面时产生的热能使材料熔化，并通过调节功率 和扫描方式使熔池保持在很高的温度, 在这种高温

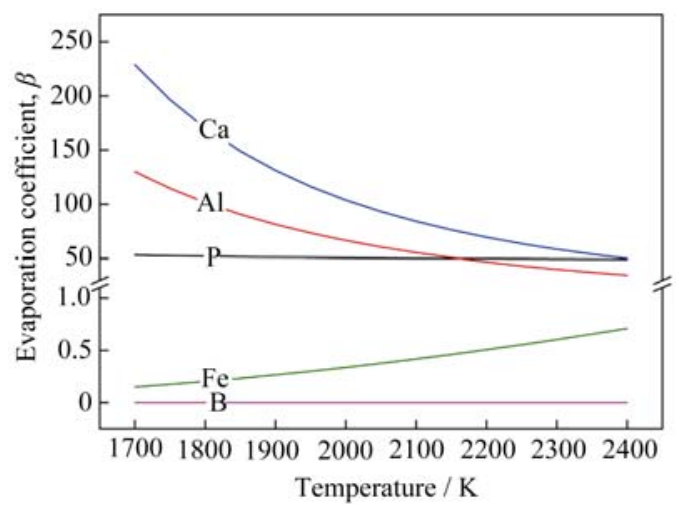

图 1 硅中主要杂质的蒸发系数与温度的关系 ${ }^{[20]}$

Fig. 1 Volatility coefficients of impurities in silicon as a function of temperature ${ }^{[20]}$ 
高真空环境下, 挥发性杂质可以从硅熔体表面蒸发 去除, 如图 2 所示 ${ }^{[21]}$ 。挥发性杂质以气体形式从 硅熔体表面挥发与液相形成气/液平衡状态, 气体 不断地在真空室内壁冷凝或被真空系统抽出炉体, 从而破坏了气/液平衡状态, 导致硅熔体中的挥发 性杂质元素不断地从熔体表面挥发出来, 达到净 化效果，而杂质被去除的程度取决于熔池的温度 以及熔炼时间 ${ }^{[22]}$ 。

电子束熔炼后获得的多晶硅锭如图 3 所示。硅 锭的表面非常光亮, 可以用肉眼分辨出晶粒的大小, 其中心部位呈现明显突起, 这是由于硅具有热缩冷 胀的热学特性, 固相体积较液相要大, 在凝固过程 中由于体积膨胀在最后凝固区域会形成凸起。硅锭 的底部比较粗粘, 这是由于水冷铜坩埚具有良好的 传热效果, 底部大量的热量被冷却水带走, 导致熔 硅接触到水冷铜坩埚后便迅速凝固。将硅锭沿中心 线切开, 可以观察到其纵截面大部分为柱状晶区, 且侧壁晶粒向中心生长。这是由于熔炼过程中硅熔 体的底部和侧壁直接与水冷铜诗埚接触, 在凝固时 会形成由顶部到底部、由侧边向中心的温度梯度, 晶粒沿着最大温度梯度的反方向生长就会形成如图 3 所示的形貌。

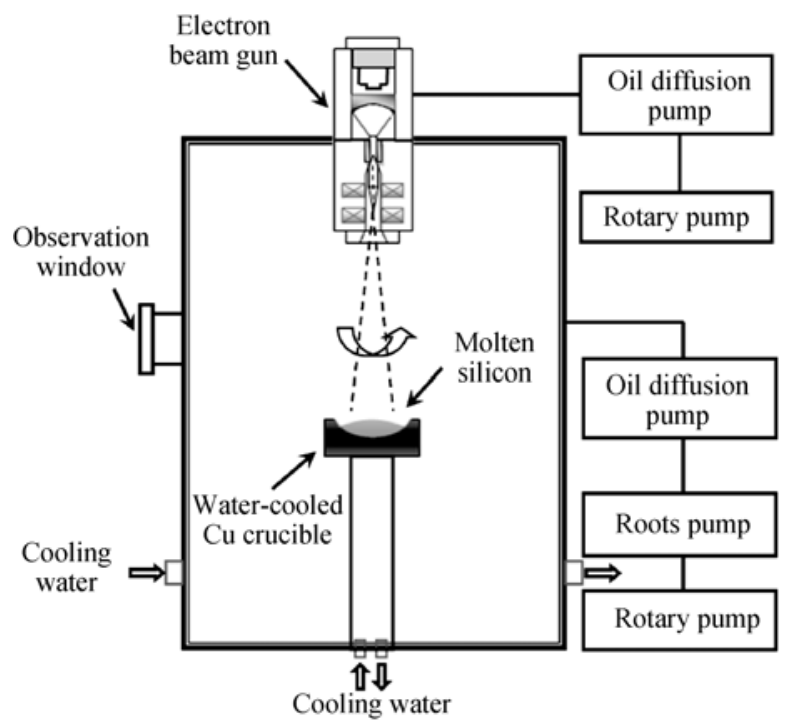

图 2 电子束熔炼多晶硅过程示意图 ${ }^{[21]}$

Fig. 2 Schematic diagram of silicon refining by electron beam melting ${ }^{[21]}$

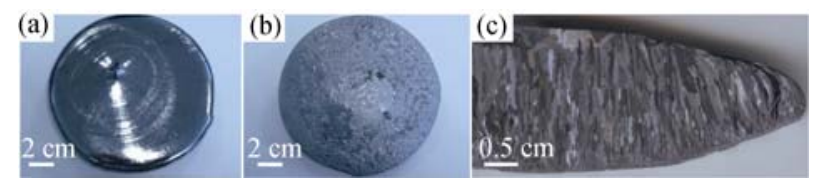

图 3 电子束熔炼制备的硅锭的宏观形貌 ${ }^{[20]}$

Fig. 3 Morphologies of electron beam melted silicon ingot ${ }^{[20]}$ (a) Surface; (b) Bottom; (c) Cross section
电子束具有能量密度高的特点, 在加热过程中 使熔池表面保持极高的温度。在同样的熔炼温度下， 饱和蒸气压比硅大的杂质元素可以有效地去除, 而 比硅的饱和蒸气压小的元素去除效果则不明显。P、 $\mathrm{Al} 、 \mathrm{Ca}$ 都是硅中的挥发性元素，电子束熔炼后去除 效果较好，熔炼 30 min 去除率即可达到 93\%、75\% 和 $89 \%$, 并且随功率增加、提纯时间延长，其去除 效果增加。另外, 电子束对 C 元素也有很好的去除 效果, 去除率达到 $90 \%{ }^{[15]}$ 。B 在 $2000 \mathrm{~K}$ 时的饱和蒸 气压只有 $10^{-2} \mathrm{~Pa}$ 的数量级, 远小于硅在此温度下的 值, 因此难以从熔硅中蒸发出来 ${ }^{[23]}$ 。

Miyake 等 ${ }^{[24]}$ 研究了低真空条件下(5 7 Pa)电子 束熔炼对硅中杂质的去除效果, 熔炼 $5 \mathrm{~min}$ 后 As 的 含量从 $0.03 \mathrm{wt} \%$ 降至 $0.1 \times 10^{-4} \mathrm{wt} \%$ 以下, 熔炼 $1 \mathrm{~h}$ 后 $\mathrm{P}$ 的含量由 $0.02 \mathrm{wt} \%$ 降至 $1 \times 10^{-4} \mathrm{wt} \%$, 并且低真空具 有与高真空相同的去除速率。随着 P、As 等杂质的 含量降低, 硅材料的电阻率显著提高 ${ }^{[25]}$ 。

Pires 等 ${ }^{[26-27]}$ 使用电子束分别熔炼了多晶硅块 料和酸洗后的多晶硅粉料, 将其纯度从 $99 \%$ 提高到 99.999\%, 结果表明电子束对两种原料都能达到预 期的提纯效果, 说明使用电子束熔炼可以避免破碎 和酸洗环节带来的高成本。同时，对杂质元素在多 晶硅锭中的分布规律进行了研究。硅熔体冷却时, 在电子束作用下内部形成了温度梯度，使其自下而 上、从边缘到中心缓慢凝固, 因此杂质元素在硅锭 中具有分凝效果, 如图 4 所示。 $\mathrm{Cu} 、 \mathrm{Al} 、 \mathrm{Ti}$ 和 $\mathrm{Fe}$ 等分凝系数远小于 1 的杂质, 会向硅锭中心和顶部 富集; 而 $\mathrm{B} 、 \mathrm{O} 、 \mathrm{P}$ 和 $\mathrm{C}$ 等分凝系数接近于 1 的杂质 在硅锭中的分布比较均匀。

Tan 等 ${ }^{[20-21,28-32]}$ 在冶金法提纯硅材料领域做了 大量的工作, 并且在国内率先开展电子束熔炼提纯 太阳能级多晶硅的研究, 深入探讨了电子束功率、 熔炼时间和凝固方式对提纯效果的影响，已经成功 地在实验室条件下将硅中磷的含量稳定地降低到 $0.1 \times 10^{-4} \mathrm{wt} \%$ 以下, Al、Ca 的去除率达到 $98 \%$, 满足 太阳能级硅材料的使用要求。同时, Tan 等 ${ }^{[33]}$ 还对硅 中 $\mathrm{O}$ 元素的去除效果进行了研究, 电子束熔炼 $10 \mathrm{~min}$ 即可将硅中的 $\mathrm{O}$ 元素从 $6.177 \times 10^{-4} \mathrm{wt} \%$ 降低到 $5.71 \times 10^{-6} \mathrm{wt} \%$ 以下，具有很高的去除效率，为多晶 硅铸锭尾料的循环再利用提供了一个崭新的思路。

\section{3 杂质去除机制}

在明确电子束熔炼对杂质的去除效果之后, 研 究人员将关注的焦点转向杂质去除机制的研究 ${ }^{[34]}$, 阐明在电子束作用下，杂质元素在熔硅中的存在状 

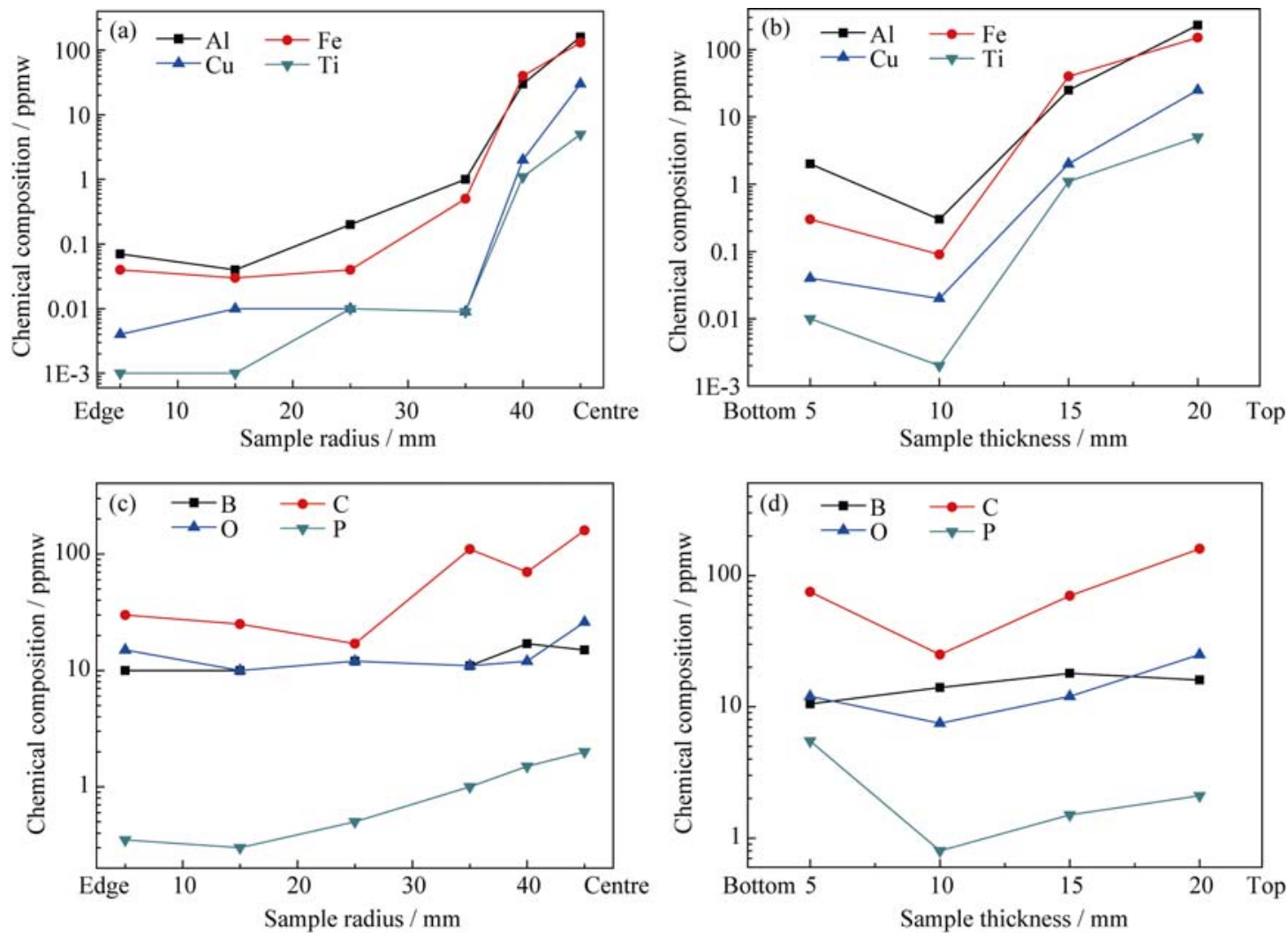

图 4 杂质在电子束熔炼制备的硅锭中的分布 ${ }^{[26]}$

Fig. 4 Distribution of impurities in electron beam melted silicon ingot ${ }^{[26]}$

态以及在熔体内部、气液界面和在气相中的运动行 为, 以期通过优化熔炼参数, 提高杂质去除效率。

\section{1 反应级数和速率常数}

根据反应动力学原理, 杂质蒸发的速率可以用 下式表示 ${ }^{[35]}$

$$
\text { Rate }=-\frac{\mathrm{d} C}{\mathrm{~d} t}=k \frac{A}{V}\left(C-C_{\mathrm{e}}\right)^{n}
$$

其中, $C$ 表示熔炼时间为 $t$ 时杂质在硅中的含量, $C_{\mathrm{e}}$ 表示最终达到平衡时杂质的含量, $A$ 是硅熔体的表 面积, $V$ 是硅熔体的体积, $n$ 即为反应级数, 系数 $k$ 被 称为反应的速率常数, 也被称为总传质系数。在实 验中, 假定杂质最终可以被完全去除, 通过其含量 与熔炼时间的关系, 来判断该种杂质的反应级数。

Peng 和 Shi ${ }^{[28-29,32]}$ 等使用几种不同的电子束功率进 行了一系列熔炼实验, 在半对数坐标下将杂质含量 与其初始含量的比值对熔炼时间作图, 发现该比值 与熔炼时间成线性关系, 表明 $\mathrm{P} 、 \mathrm{Al} 、 \mathrm{Ca}$ 三种杂质 的去除遵循一级反应动力学, 该结论与 Ikeda ${ }^{[15]}$ 、 Hanazawa ${ }^{[36]}$ 以及 Kemmotsu ${ }^{[37]}$ 等研究一致。此外, Ikeda 等还发现 C 元素的去除也遵循一级反应动力 学, 因此可以认为, 在电子束熔炼的温度范围内, 这些杂质从硅熔体表面的蒸发是以单原子的方式进
行的。

速率常数可以直观地表征反应进行的快慢, 通过上述归一化杂质含量与熔炼时间关系图中所 拟合直线的斜率，可以获得在不同实验条件下杂 质去除的速率常数, 如图 5 所示。从图 5 可以看 出, 杂质蒸发去除反应的速率常数随着温度的升 高而增大，说明熔炼功率越大，熔体温度越高, 杂质去除得越快。

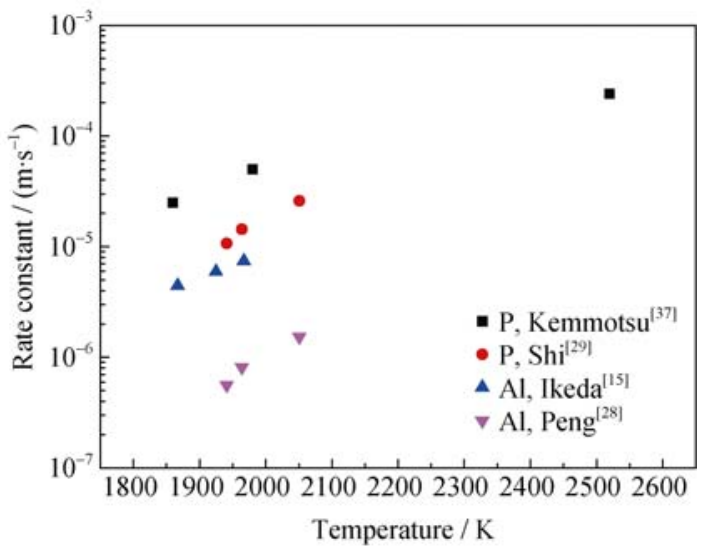

图 5 硅中杂质蒸发的速率常数与温度的关系 ${ }^{[15,28-29,37]}$

Fig. 5 Rate constant of impurity evaporation from silicon as a function of temperature ${ }^{[15,28-29,37]}$ 


\section{2 杂质去除的速率控制步骤}

电子束熔炼提纯过程中, 熔体中的挥发性元 素从熔体内部向气相传输, 并在远离熔体界面处 冷凝，如图 6 所示。该过程主要通过以下几个步 骤进行：(1)杂质元素从熔体内部迁移到液相边界 层的传质过程; (2)从液相边界层扩散到熔体表面 的传质过程; (3)在熔体表面蒸发进入气相的过程; (4)在气相中的传质过程; (5)在真空室内壁冷凝或 被真空系统带走。

这些步骤中的一个或多个的组合会成为杂质去 除的速率控制步骤, 主要取决于电子束熔炼的特征 和实验条件。步骤(1)通常不是速率控制步骤, 这是 由于在电子束熔炼过程中, 硅熔体内存在很大的温 度梯度, 导致熔体流动加剧, 使杂质在熔体内部始 终分布均匀; 挥发到气相中的杂质最终在冷凝表面 上凝结, 或通过真空系统排出, 只要达到气态物质 冷凝的最低热力学条件, 气相的冷凝过程即可迅速 进行, 因此步骤(5)也不是速率控制步骤。综上可知, 步骤(2)至步骤(4)中的一个或多个过程会成为杂质 去除的速率控制步骤。

Hanazawa 等 ${ }^{[36]}$ 研究了电子束熔炼过程中杂质 $\mathrm{P}$ 的去除行为, 认为 $\mathrm{P}$ 在熔体表面的蒸发是其去除的速 率控制步骤, 因此通过增加电子束功率提高熔体表面 的温度可以加快 P 的去除速率。Peng 和 Shi ${ }^{[28-29,32] \text { 等 }}$ 通过建立真空熔炼过程中元素蒸发传输模型来判 断杂质元素去除的速率控制步骤, 认为杂质 $\mathrm{P}$ 在硅 熔体内部扩散传质和在硅熔体表面的自由蒸发共 同控制其蒸发去除过程, 而杂质 $\mathrm{Al}$ 和 $\mathrm{Ca}$ 在硅熔体 内部扩散传质步骤是其蒸发去除过程的速率控制 步骤。

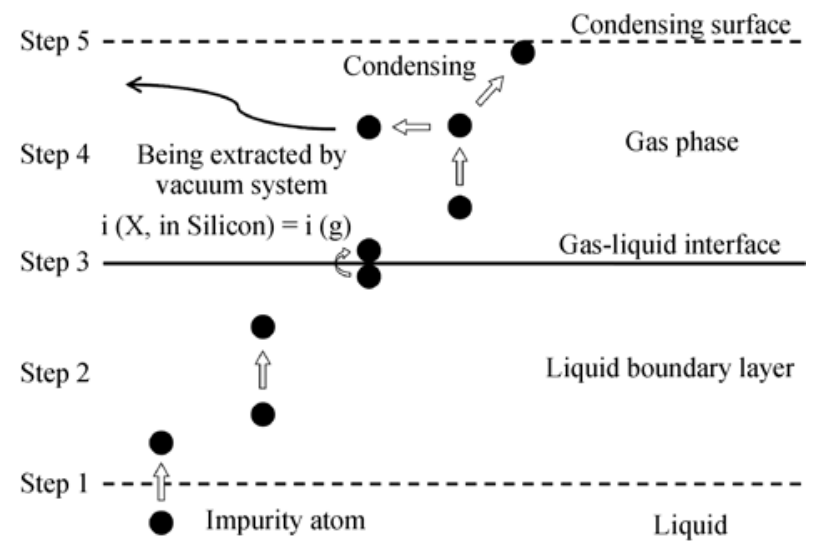

图 6 挥发性杂质从硅熔体到气相的传输过程 ${ }^{[29]}$

Fig. 6 Schematic diagram of the migration process of impurity atom during different steps ${ }^{[29]}$

\section{4 电子束熔炼提纯多晶硅的研究现状}

电子束熔炼技术是一种去除硅中挥发性杂质的 有效方法，成为治金法制备太阳能级多晶硅的关键 环节之一，已被应用于工业生产中，取得了良好的 效果。然而，该技术尚存在能量利用率偏低、与冶 金法前后环节之间衔接性差等不足，成为制约其大 规模产业化应用的瓶颈。因此, 当前研究重点在于 充分发挥电子束技术的潜力, 开发高效、低能耗的 电子束熔炼提纯技术。

\section{1 电子束熔炼过程的数值模拟}

电子束熔炼是一种能量高度集中的表面加热方 式, 同时使用水冷铜坩埚作为熔炼容器, 导致硅熔 体内部存在相当大的温度梯度, 熔炼过程中大量的 热量被冷却系统带走, 造成能量的利用率偏低。并 且, 熔池的温度还是影响杂质去除的主要因素, 但 在电子束熔炼条件下，对熔池温度的测量不易实施， 因此有必要引入计算模拟的方法对整个熔炼过程进 行仿真, 为优化熔炼参数、降低能耗提供理论依据。

Maijer 等 ${ }^{[38]}$ 建立了电子束熔炼多晶硅的传热学 模型, 预测了熔炼过程中的温度变化, 与实验结果 符合得较好, 并进一步通过该模型计算了冷却过程 中铸锭中的残余应力。Kraze 等 ${ }^{[39]}$ 将低频横向磁场 引入到电子束熔炼的过程中, 并通过有限元方法模 拟了磁场的引入对硅熔体的温度和流动行为的影响, 结果表明, 磁场的引入有助于增加熔体自身的搅动, 减小凝壳的厚度。Choi 等 ${ }^{[40]}$ 研究了不同电子束扫描 方式对熔池温度和形貌的影响。

Wen 等在对硅-铜接触热阻研究的基础上 ${ }^{[41]}$, 建立了电子束熔炼去除硅中磷杂质的数值模型, 提 出了参数优化的准则 ${ }^{[42]}$ 。结果表明, 熔池表面的温 度与电子束功率近似成线性关系, 并随着功率的增 加和扫描半径的减小而升高。对于 $0.5 \mathrm{~kg}$ 的硅料, P 含量从 $1.44 \times 10^{-2} \mathrm{wt} \%$ 降低到 $1 \times 10^{-5} \mathrm{wt} \%$ 所消耗的能 量随着扫描半径的减小和功率的增加而降低, 最佳 参数选择为功率 $23.4 \mathrm{~kW}$ 、扫描半径 $3.39 \mathrm{~cm}$, 如图 7 所示。

\section{2 小功率、浅熔池熔炼方式的提出}

电子束熔炼的原料来源于前端的定向凝固, 需 要对定向凝固的铸锭进行破碎, 前后环节之间衔接 性差, 增加了工艺流程。Jiang 等 ${ }^{[43-45]}$ 结合电子束熔 炼和凝固的特征, 提出了一种小功率、浅熔池的熔 炼技术, 即电子束烛光熔炼技术, 利用电子束圆形 波扫描所形成的具有最大表面积和最浅熔池深度的 


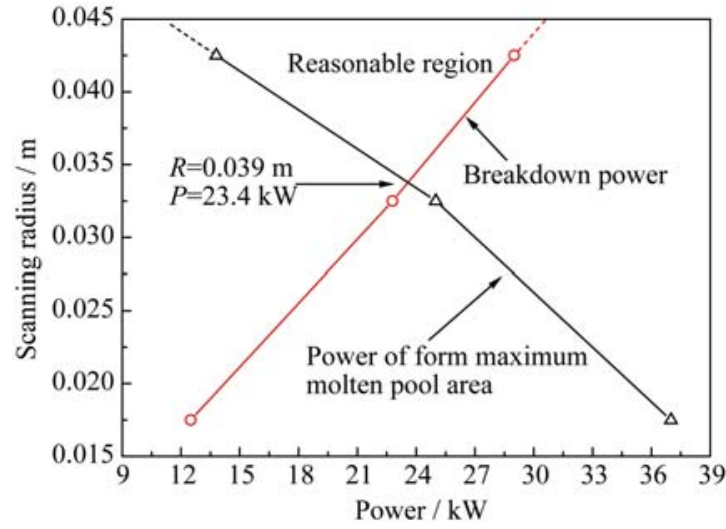

图 $70.5 \mathrm{~kg}$ 的硅在电子束熔炼时的参数优化结果 ${ }^{[42]}$

Fig. 7 The optimum value of melting parameters for $0.5 \mathrm{~kg}$ silicon by electron beam melting ${ }^{[42]}$

临界熔池状态，采用周期性熔炼的方式逐步将定向 凝固的铸锭全部熔炼, 如图 8 所示。熔炼过程中, 熔 池远离水冷铜坩埚, 电子束的能量得到了充分的利 用, 可以在低能量下熔炼较大质量的铸锭, 减小了 硅的损失率。

研究结果表明, 与传统的电子束熔炼方式相比, 在相同的时间或功率下，该方法拥有较高的杂质去 除效率。电子束功率为 $6 \mathrm{~kW}$ 的实验中, 熔炼时间为 $300 \mathrm{~s}$ 时, 杂质磷的去除率达到了 $60 \%$ 左右，而在传 统的电子束熔炼方式下, 得到相同的去除率需要在 $15 \mathrm{~kW}$ 下熔炼 $300 \mathrm{~s}$, 或在 $9 \mathrm{~kW}$ 下熔炼 $600 \mathrm{~s}$ 。使用 该技术可以实现电子束高效、低能耗去除杂质, 并 与前端定向凝固技术有效衔接，减少了铸锭破碎环 节，大幅度提高生产效率。

\section{3 电子束熔炼技术与定向凝固技术的耦合}

硅中的某些杂质同时具有蒸发和分凝两种特

征：其饱和蒸气压远远大于硅的饱和蒸气压，在高 温、高真空的条件下可以被蒸发去除; 其在硅中的 分凝系数远远小于 1 , 可以通过定向凝固的方式被 去除。传统的电子束熔炼方式仅仅利用了电子束高 温、高真空的特点去除挥发性的杂质，并没有考虑 硅凝固时杂质的一些行为，没有充分发挥电子束 熔炼的优势。

我们前期研究发现, 通过电子束降束诱导的方 式可以实现硅熔体的定向凝固, 如图 9 所示, 且采 用指数型的功率下降方法, 硅熔体会以近似恒定的 速率凝固 ${ }^{[46]}$ 。在对杂质蒸发和分凝耦合理论进一步 完善的基础上 ${ }^{[47-48]}$, 研究了杂质在电子束诱导定向 凝固铸锭中的分布。这类杂质在铸锭中具有明显的 分凝效应，分凝作用将杂质推到硅熔体中，提高硅 熔体中杂质浓度，促进蒸发作用下杂质的去除效果， 相比于完全的蒸发提纯过程，当总时间一致时，电

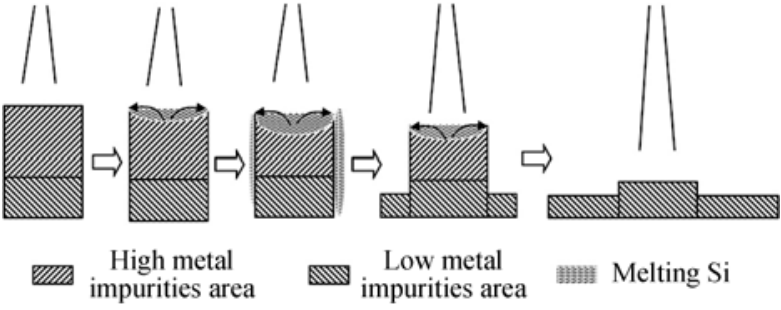

图 8 小功率、浅熔池熔炼方式示意图 ${ }^{[44]}$

Fig. 8 Process of electron beam melting with low power and shadow melten pool ${ }^{[44]}$
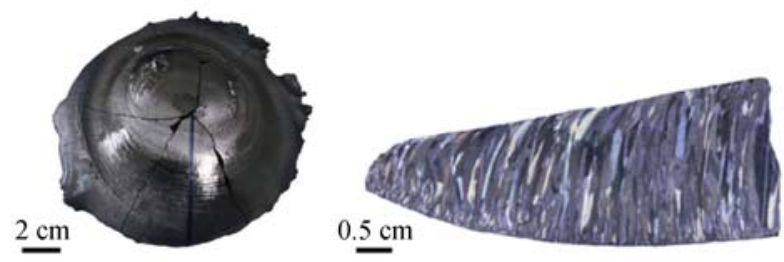

图 9 电子束诱导多晶硅定向凝固铸锭 ${ }^{[46]}$

Fig. 9 Directional solidified silicon ingot induced by electron beam $^{[46]}$

子束熔炼与降束凝固的过程对杂质的去除效果要优 于完全蒸发提纯过程，同时电子束熔炼与降束凝固 过程降低了能量的消耗 ${ }^{[49]}$ 。

Yuge 等 $^{[50]}$ 在电子束设备内部设置一个熔炼坩 埚和一个凝固坩埚。熔炼坩埚中的硅被加热至全部 熔化并保持在较高的温度，在此过程中，挥发性杂 质通过硅熔体表面被蒸发去除; 之后，硅熔体进入 到凝固坩埚中, 在电子束和水冷系统的共同作用下 实现定向凝固，在此过程中，分凝系数小的杂质富 集到铸锭顶部并最终被去除。用这种方式将硅中的 $\mathrm{Fe} 、 \mathrm{Al}$ 和 $\mathrm{Ca}$ 分别去除到 $1 \times 10^{-4} \mathrm{wt} \%$ 以下，通过降低 凝固速率、增加电子束功率、使用纯度更高的原料、 增加熔池深度或者减小溶质扩散层厚度等方式可以 获得更好的提纯效果。

Lee 等 ${ }^{[51]}$ 使用同样的熔炼方法并将凝固坩埚改为 可下降式, 将硅中挥发性杂质 $\mathrm{Al} 、 \mathrm{Ca} 、 \mathrm{Mg} 、 \mathrm{Mn}$ 以及 分凝性杂质 $\mathrm{Fe} 、 \mathrm{Ti} 、 \mathrm{Ni} 、 \mathrm{Cr} 、 \mathrm{Cu}$ 去除到 ICP-OES 的 检测限以下，得到了纯度为 $99.999 \%$ 的多晶硅铸锭， 且出成率达到 $90 \%$ 。随后, Lee 等 ${ }^{[52]}$ 又对这种熔炼方 式做了改进, 将熔炼坩埚去掉, 直接在凝固坩埚中实 现熔炼和凝固过程，并探讨了熔炼后期电子束功率控 制对熔体内部热流方向，进而对凝固组织和杂质富集 区的影响。结果表明，熔炼后期以恒定速率下降的电 子束功率可以减小熔体中的温度梯度, 从而有效地减 少晶界的体积和杂质在晶界上的分凝，通过这种熔炼 方式，可以将纯度为 $99 \%$ 的冶金级硅一次性提纯到 99.999\%以上，同时获得了较高的铸锭出成率。 
此外, Mei 等 ${ }^{[53]}$ 将电子束技术和区域熔炼结合 起来, 使用电子束作为移动热源实现了水平区域熔 炼, 将纯度为 99\%的多晶硅提纯到纯度为 99.999\%, 并研究了区熔速率对提纯效果的影响。结果表明, 这种方法对除 B 之外的大部分杂质都有效, 且较慢 的区熔速率能够获得更好的提纯效果。

\section{5 总结与展望}

电子束熔炼提纯技术用于制备太阳能级多晶硅, 能够有效地去除其中的挥发性杂质元素和分凝系数 小的杂质元素, 并且已经大规模地应用于工业生产 中, 取得了显著的成效。前述研究主要集中在对杂质 去除效果的评估以及对杂质在熔炼过程中的运动行 为和去除机制的探索, 为电子束熔炼的应用提供理 论支撑。当前, 电子束熔炼技术还存在许多亟待解决 的问题, 需要从以下几个方面解决:

1)电子束熔炼多相多物理场复杂系统的计算机 模拟。电子束熔炼是一个涉及到物理、化学反应的 复杂非稳态过程, 包含了发生在液相、气相和气液界 面的能量传输、动量传输和质量传输三个过程以及 在气液界面的溶解和脱气反应。在前期对电子束熔 炼温度场进行数值模拟的基础上, 进一步考虑温度 场与流场的交互作用以及杂质的动态分布, 深入理 解杂质的去除机制并对杂质输运进行控制。

2)探索效率更高、能耗更低的电子束熔炼方式。 传统的电子束熔炼方式是将材料完全熔化形成熔池, 保持在较高的温度长时间熔炼, 使熔体内部的杂质 有充分的时间扩散到表面。然而, 长时间熔炼导致大 量的能量被冷却系统带走, 基体材料的挥发损失也 越大, 综合能量利用率不高。探索新的电子束熔炼方 法和工艺, 降低综合能耗成为发展高效、低能耗电子 束熔炼技术的一个途径。

电子束熔炼技术在太阳能级多晶硅的制备方面 具有巨大的优势和广阔的市场应用前景, 以上问题 的解决对于打破制约该技术大规模推广的瓶颈会发 挥至关重要的作用。随着对电子束熔炼提纯过程的 理解不断深入, 通过引入多相多物理场复杂系统的 计算机模拟方法, 发展高效率、低能耗的电子束熔炼 新方法, 使电子束熔炼技术的应用不断得到推进, 可以进一步降低太阳能级多晶硅的制造成本, 同时 为电子束熔炼技术应用空间的拓展开辟道路。

\section{参考文献:}

[1] SWANSON R M. Photovoltaics power up. Science, 2009,
324(5929): 891-892.

[2] POWELL D M, WINKLER M T, CHOI H J, et al. Crystalline silicon photovoltaics: a cost analysis framework for determining technology pathways to reach baseload electricity costs. Energy Environ. Sci., 2012, 5(3): 5874-5883.

[3] COLETTI G. Sensitivity of state-of-the-art and high efficiency crystalline silicon solar cells to metal impurities. Prog. Photovoltaics, 2013, 21(5): 1163-1170.

[4] TYAGI V V, RAHIM N A A, RAHIM N A, et al. Progress in solar PV technology: research and achievement. Renew. Sust. Energ. Rev., 2013, 20: 443-461.

[5] BUONASSISI T, ISTRATOV A A, MARCUS M A, et al. Engineering metal-impurity nanodefects for low-cost solar cells. Nat. Mater., 2005, 4(9): 676-679.

[6] HUDELSON S, NEWMAN B K, BERNARDIS S, et al. Retrograde melting and internal liquid gettering in silicon. Adv. Mater., 2010, 22(35): 3948-3953.

[7] PIZZINI S. Towards solar grade silicon: challenges and benefits for low cost photovoltaics. Sol. Energy Mater. Sol. Cells, 2010, 94(9): 1528-1533.

[8] CIFTJA A. Rrefining of solar cell silicon through metallurgical routes. JOM, 2012, 64(8): 933-934.

[9] CORTES A D S, SILVA D S, VIANA G A, et al. Solar cells from upgraded metallurgical-grade silicon purified by metallurgical routes. J. Renew. Sustain. Energy, 2013, 5(2): 123-129.

[10] YU W Z, MA W H, LV G Q, et al. Low-cost process for silicon purification with bubble adsorption in Al-Si melt. Metall. Mater. Trans. B, 2014, 45(4): 1573-1578.

[11] FANG M, LU C H, HUANG L Q, et al. Multiple slag operation on boron removal from metallurgical-grade silicon using $\mathrm{Na}_{2} \mathrm{O}-\mathrm{SiO}_{2}$ slags. Ind. Eng. Chem. Res., 2014, 53(30): 12054-12062.

[12] CHOUDHURY A, HENGSBERGER E. Electron beam melting and refining of metals and alloys. ISIJ Int., 1992, 32(5): 673-681.

[13] BAKISH R. The substance of a technology: Electron-beam melting and refining. JOM, 1998, 50(11): 28-30.

[14] CASENAVE D, GAUTHIER R, PINARD P. A study of the purification process during the elaboration by electron bombardment of polysilicon ribbons designed for photovoltaic conversion. Sol. Energy Mater., 1981, 5(4): 417-423.

[15] IKEDA T, MAEDA M. Purification of metallurgical silicon for solar-grade silicon by electron beam button melting. ISIJ Int., 1992, 32(5): 635-642.

[16] KATO Y, HANAZAWA K, BABA H, et al. Purification of metallurgical grade silicon to solar grade for use in solar cell wafers. Tetsu To Hagane-J. Iron Steel Inst. Jpn., 2000, 86(11): 9-16.

[17] YUGE N, ABE M, HANAZAWA K, et al. Purification of metallurgical-grade silicon up to solar grade. Prog. Photovoltaics, 2001, 9(3): 203-209.

[18] MITRASINOVIC A M, D'SOUZA R, UTIGARD T A. Impurity removal and overall rate constant during low pressure treatment of liquid silicon. J. Mater. Process. Technol., 2012, 212(1): 78-82.

[19] SAFARIAN J, TANGSTAD M. Vacuum refining of molten silicon. Metall. Mater. Trans. B, 2012, 43(6): 1427-1445.

[20] 王 强. 电子束熔炼提纯冶金级硅工艺研究. 大连: 大连理工大 学硕士学位论文, 2010.

[21] WANG Q, DONG W, TAN Y, et al. Impurities evaporation from metallurgical-grade silicon in electron beam melting process. Rare Metals, 2011, 30(3): 274-277.

[22] SUN J L, ZHANG J, WANG H W, et al. Purification of metallur- 
gical grade silicon in an electron beam melting furnace. Surf. Coat. Technol., 2013, 228: S67-S71.

[23] LIU T, DONG Z Y, ZHAO Y W, et al. Large scale purification of metallurgical silicon for solar cell by using electron beam melting. J. Cryst. Growth, 2012, 351(1): 19-22.

[24] MIYAKE M, HIRAMATSU T, MAEDA M. Removal of phosphorus and antimony in silicon by electron beam melting at low vacuum. J. Jpn. Inst. Met., 2006, 70(1): 43-46.

[25] OSOKIN V A, SHPAK P A, ISHCHENKO V V, et al. Electronbeam technology for refining polycrystalline silicon to be used in solar power applications. Metallurgist, 2008, 52(1/2): 121-127.

[26] PIRES J C S, BRAGA A F B, MEI P R. Profile of impurities in polycrystalline silicon samples purified in an electron beam melting furnace. Sol. Energy Mater. Sol. Cells, 2003, 79(3): 347-355.

[27] PIRES J C S, OTUBO J, BRAGA A F B, et al. The purification of metallurgical grade silicon by electron beam melting. J. Mater. Process. Technol., 2005, 169(1): 16-20.

[28] PENG X, DONG W, TAN Y, et al. Removal of aluminum from metallurgical grade silicon using electron beam melting. Vacuum, 2011, 86(4): 471-475.

[29] SHI S, DONG W, PENG X, et al. Evaporation and removal mechanism of phosphorus from the surface of silicon melt during electron beam melting. Appl. Surf. Sci., 2013, 266: 344-349.

[30] TAN Y, GUO X L, SHI S, et al. Study on the removal process of phosphorus from silicon by electron beam melting. Vacuum, 2013, 93: $65-70$.

[31] 彭 旭. 电子束熔炼冶金硅中杂质蒸发行为研究. 大连: 大连理 工大学硕士学位论文, 2011.

[32] 姜大川. 电子束熔炼提纯多晶硅的研究. 大连: 大连理工大学 博士学位论文, 2012.

[33] ASGHAR H M N U K, TAN Y, SHI S, et al. Removal of oxygen from silicon by electron beam melting. Appl. Phys. A, 2014, 115(3): 753-757.

[34] SASAKI H, KOBASHI Y, NAGAI T, et al. Application of electron beam melting to the removal of phosphorus from silicon: toward production of solar-grade silicon by metallurgical processes. $A d v$. Mater. Sci. Eng., 2013, 2013: 857196-1-8.

[35] SAFARIAN J, TANGSTAD M. Kinetics and mechanism of phosphorus removal from silicon in vacuum induction refining. High Temp. Mater. Process., 2012, 31(1): 73-81.

[36] HANAZAWA K, YUGE N, KATO Y. Evaporation of phosphorus in molten silicon by an electron beam irradiation method. Mater. Trans., 2004, 45(3): 844-849.

[37] KEMMOTSU T, NAGAI T, MAEDA M. Removal rate of phosphorus from molten silicon. High Temp. Mater. Process., 2011, 30(1/2): $17-22$

[38] MAIJER D M, IKEDA T, COCKCROFT S L, et al. Mathematical modeling of residual stress formation in electron beam remelting and refining of scrap silicon for the production of solar-grade silicon. Mater. Sci. Eng. A, 2005, 390(1/2): 188-201.

[39] KRAZE A, MUIZNIEKS A, BERGFELDS K, et al. Reduction of silicon crust on the crucible walls in silicon melt purifying processes with electron beam technology by low-frequency travelling magnetic fields. Magnetohydrodynamics, 2011, 47(4): 369-383.

[40] CHOI S H, JANG B Y, LEE J S, et al. Effects of electron beam patterns on melting and refining of silicon for photovoltaic applications. Renew. Energy, 2013, 54: 40-45.

[41] WEN S T, TAN Y, SHI S, et al. Thermal contact resistance between the surfaces of silicon and copper crucible during electron beam melting. Int. J. Therm. Sci., 2013, 74: 37-43.

[42] TAN Y, WEN S T, SHI S, et al. Numerical simulation for parameter optimization of silicon purification by electron beam melting. Vacuum, 2013, 95: 18-24.

[43] JIANG D C, TAN Y, SHI S, et al. Removal of phosphorus in molten silicon by electron beam candle melting. Mater. Lett., 2012, 78: 4-7.

[44] JIANG D C, TAN Y, SHI S, et al. Evaporated metal aluminium and calcium removal from directionally solidified silicon for solar cell by electron beam candle melting. Vacuum, 2012, 86(10): 1417-1422.

[45] JIANG D C, TAN Y, SHI S, et al. Research on new method of electron beam candle melting used for removal of $\mathrm{P}$ from molten Si. Mater. Res. Innov., 2011, 15(6): 406-409.

[46] TAN Y, SHI S, GUO X L, et al. Effect of cooling rate on solidification of electron beam melted silicon ingots. Vacuum, 2013, 89: $12-16$.

[47] TAN Y, REN S Q, SHI S, et al. Removal of aluminum and calcium in multicrystalline silicon by vacuum induction melting and directional solidification. Vacuum, 2014, 99: 272-276.

[48] JIANG D C, REN S Q, SHI S, et al. Phosphorus removal from silicon by vacuum refining and directional solidification. J. Electron. Mater, 2014, 43(2): 314-319.

[49] JIANG D C, SHI S, TAN Y, et al. Research on distribution of aluminum in electron beam melted silicon ingot. Vacuum, 2013, 96: 27-31.

[50] YUGE N, HANAZAWA K, KATO Y. Removal of metal impurities in molten silicon by directional solidification with electron beam heating. Mater. Trans., 2004, 45(3): 850-857.

[51] LEE J K, LEE J S, JANG B Y, et al. Directional solidification behaviors of polycrystalline silicon by electron-beam melting. Jpn. J. Appl. Phys., 2013, 52(10): 10MB09-1-5.

[52] LEE J K, LEE J S, JANG B Y, et al. Impurity segregation behavior in polycrystalline silicon ingot grown with variation of electron-beam power. Jpn. J. Appl. Phys., 2014, 53(8): 08NJ05-1-6.

[53] MEI P R, MOREIRA S P, CARDOSO E, et al. Purification of metallurgical silicon by horizontal zone melting. Sol. Energy Mater. Sol. Cells, 2012, 98: 233-239. 